\title{
Position control of a ferromagnetic micro-particle in a dry environment
}

\author{
Soukeyna Bouchebout ${ }^{1}$, Aude Bolopion ${ }^{2}$, Michaël Gautier ${ }^{2}$ and Stéphane Régnier ${ }^{1}$
}

\begin{abstract}
Most of current wireless micro-robotic systems evolve in liquids, as the predominance, at this scale, of surface adhesion makes it hard to control these systems in dry environments. The purpose of this article is to propose a method to improve wireless micro-devices performances in the air, by focusing on the workspace substrate. In this work, a magnetically actuated micro-particle is described and a strategy to limit the surface forces is presented. The micro-device behavior is studied and compared on five different surfaces. We demonstrate that reducing the electrical resistivity and the roughness of the surface helps improving the particle actuation occurrence. Furthermore, to validate the proposed approach, the particle is positioned with high precision using a closed loop control based on visual feedback. The micro-particle response is identified using a statistical approach over a large microparticle response database.
\end{abstract}

\section{INTRODUCTION}

The new field of untethered micro/nano sized devices presents promising applications in several fields. For example, in biomedicine inside micro-fluidic chips [1], in medicine for mini invasive surgery devices [2] and targeted drug delivery, as well as in advanced manufacturing to assemble micro-components or to perform mechanical characterization [3]. Untethered micro-devices benefit from their small size to fit in clustered environment and perform tasks on micro-objects. The low inertia of these devices allows high velocities, hence they can carry out many actions in a short time.

However, the reduced size of the untethered submillimeter devices makes it currently impracticable to be embedded with energy sources or sensors. Off-board power methods can be used at this scale. Major progresses have been achieved in this area. Electrostatic effect [4] is used to wirelessly power scratch drive devices. However, this method requires specific surfaces to operate on. Otherwise, the dielectrophoresis actuation [5] uses the dielectric forces to create motion but needs a high voltage. Moreover, electromagnetism is widely used as an external energy source. For instance, the Magnetically driven MicroTool (MMT) [1] [6] uses permanent magnets as a power source and presents good results in manipulating biological cells inside microfluidic channels. Swimming

\footnotetext{
*This work has been supported by the Labex ACTION project (contract "ANR-11-LABX-01-01"), the French RENATECH network and its FEMTO-ST technological facility and the French Agence Nationale de la Recherche, through the LEMA project (contract ANR 12 BS03 007 01)

${ }^{1} \mathrm{~S}$. Bouchebout · S. Régnier are with the Institut des Système Intelligents et de Robotique (ISIR), Université Pierre et Marie Curie/CNRS UMR-7222, BC 173, 4 Place Jussieu, 75005 Paris, France bouchebout, regnierdisir.upmc. fr

${ }^{2}$ A. Bolopion - M. Gautier are with the FEMTO-ST Institute, UFC, ENSMM, UTBM, CNRS UMR-6174, 24 rue Alain Savary, 25000 Besançon, France aude.bolopion@ femto-st. fr
}

micro-robots [7] [8] [9] operate using magnetic actuation as a remote propulsion in a liquid environment, hence they are well suited for in vivo and in vitro applications. Besides, other mobile micro-devices employ electromagnets for actuation [10][11][12]. Therefore, the magnetic field is well suited to remotely actuate micro-devices. Furthermore, to perform micro-assembly or micro-manipulation tasks, the control of the untethered devices is required at high speed and high precision. A closed/open loop control is based on a position/velocity/acceleration feedback. However, at this scale, embedding accelerometers or sensors is hard to achieve. To overcome this issue, image based detection using cameras [11] is widely used; as it offers, though with a limited detection field, a high speed tracking at low cost. Other localization methods can be employed like fluoroscopy, MRI (magnetic resonance imaging) [13] [14], etc.

In addition, at the micro-scale, adhesion effects due to capillary, electrostatic, van der Waals, etc., dominate the physics [15] in liquids and, in particular, dry environments. Most magnetic based systems [10][16] evolve in a liquid environment. However, in a liquid, the micro-devices velocity is reduced due to the drag force which is proportional to the device speed [17]. In contrast, micro-robotic systems operating in the air benefit from high dynamics but suffer from frequent adhesions to the surface. To avoid this issue, wireless micro-devices use, beside the magnetic actuation, the electrostatic effect [18]: the interdigitaded electrodes in an engineered surface induce a charge separation in the micro-device metallic part and therefore regulate the frictional forces. Also, the piezoelectric properties [19] can be employed to engender vibrations to a micro-particle by a successive distortion of the sample, thus limiting the adhesion to surface. Although, these methods require high voltages and specific surfaces that increase the production costs. Another solution to reduce the surface adhesion is to focus on the workspace substrate. A particular material and texture of the surface are used in [15] to perform the grasping of micro-spheres in a dry environment. The limitation of the surface adhesion is important to obtain a reliable, fast and precise control of magnetic actuated micro-devices in the air.

This article focuses on the untethered magnetically actuated micro-tool, evolving in the air. Currently, the repeatability of the micro-particle displacement in the air is insufficient for reliable control. The challenge in actuating and controlling micro-devices in a dry environment is to reduce the surface adhesion. The approach in this article relies on the substrate engineering. Using micro-fabrication processes, the surface roughness is controlled, and the effect of the substrate material and surface finish on the particle 
adhesion to the substrate is addressed. We demonstrate that the electrical properties and the substrate finish are important and enhances the particle performances in the air, beside, it enables controlling the system at high precision using a simple closed loop control. The control of the particle position is a vision based closed-loop, synthesized upon the particle response characterization using a statistical approach over a large experimental database.

The magnetic actuation in dry environments and its main challenges are presented in the second section. A solution to address the adhesion issue is proposed in the third section. The control of a magnetic actuation based system is approached in the fourth section. The final section concludes and provides directions for future works.

\section{THE UNTETHERED MICRO-ROBOTIC SYSTEM}

'Untethered' means for a micro-robotic system that its micro-end effector is wirelessly manipulating objects and evolving in the environment. In this work, vision based detection methods are used to locate the manipulator and the magnetic field is employed to actuate the end effector (also called micro-particle). The micro-robotic system is described in this section.

\section{A. Particle micro-fabrication}

To manipulate objects sized between $10-100 \mu \mathrm{m}$, a tool of a size greater than the object's size is needed, i.e from 200 to $500 \mu \mathrm{m}$. In order to produce particles of such size, microfabrication processes are used. In addition, the magnetic actuation requires a non-zero internal magnetization endeffector, for example a ferromagnetic material, as it offers the highest magnetization. The nickel is suited in this case, as it is commonly used in micro-fabrication processes.

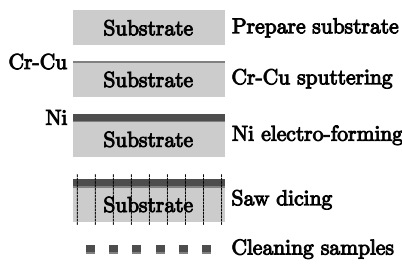

(a) Micro-fabrication flowchart.

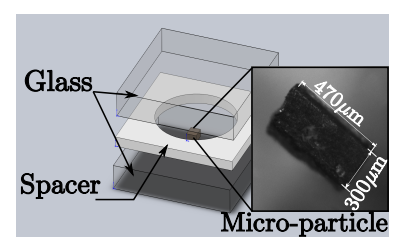

(b) The particle workspace is limited by two surfaces separated by a spacer.
Fig. 1: Fabrication process flow of a Ni micro-particle and assembly of the arena enclosing a $470 \times 300 \mu \mathrm{m}^{2}$ microparticle.

The micro-fabrication flowchart, Fig. 1(a), firstly consists in sputtering a $\sim 450 \mathrm{~nm} \mathrm{Cu}-\mathrm{Cr}$ layer on a not conductive sacrificial substrate to plate the substrate. The plating is important for the next step, the electro-forming which requires a conductive substrate. Secondly, a $\sim 25 \mu \mathrm{m}$ Ni layer is deposited on the substrate. Finally, the substrate is saw-diced into tiny rectangular samples, the resulting strain separates the Ni from the substrate.
Given that the environment disturbances (dust, humidity) are predominant at the micro-scale, the micro-particle is enclosed in an arena, Fig. 1(b). The arena contains two layers separated by a patterned spacer, which defines the shape and dimension of the micro-particle workspace. To allow the camera detection, the top layer of the arena is transparent. Besides, the micro-particle lays on the bottom layer of the arena. For preliminary experiments in this section, a glass surface is used as a substrate. Throughout the next sections, the surface is processed to properly determine the effect of the surface finish and electrical properties on the particle behavior.

\section{B. Magnetic actuation}

To wirelessly actuate the micro-particle, an external energy source is used: the magnetic field. In order to control the produced magnetic field, electromagnets are employed. Moreover, to guarantee 3DOF (Degrees of Freedom) to the micro-particle -two translations and a rotation-, four orthogonal electromagnets are suitable. The arena, where the micro-particle lies, is placed at the center of the coils, as shown in Fig. 2. The coils are actuated, sequentially, by applying an electric current I.

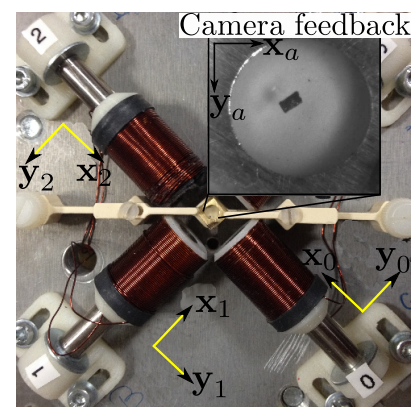

Fig. 2: Four orthogonal coils surrounding an arena, which is in the plane defined by the coils axes.

As the particle is evolving in a dry environment, the surface forces are predominant. Therefore, a strong magnetic field is required. The produced magnetic field depends on the applied current, the electromagnet dimension and geometry (turns number, coil length etc.). In order to increase the magnetic field while reducing the coils size $(170 \mathrm{~mm}$ length, 700 turns), the current is increased, iron cores are added to the coils and the electromagnets are brought as close as possible to the arena. Moreover, to prevent the coils from heating, an impulse based current signal $(h[\mathrm{~ms}]$ is the signal width and I[ampere] is the impulse intensity) is used. As for the particle detection, the image processing of the camera's frame is used to measure the particle center of gravity position ${ }^{a} \mathbf{P}_{m}=\left[{ }^{a} x_{m}{ }^{a} y_{m}\right]$ in the arena frame of reference $\mathbf{R}_{a}=\left\{\mathbf{x}_{a} \mathbf{y}_{a}\right\}$.

In this work, the displacement of the micro-particle is due to an applied magnetic force $\mathbf{F}_{m}$, though it could be based on the magnetic torque as in [11]. Therefore, the particle experiences surface forces $\mathbf{F}_{\mathrm{s}}$ and its weight, beside the magnetic force. Regarding $\mathrm{m}$ the mass of the particle, the 
equation of motion is as follow:

$$
\mathbf{F}_{\mathrm{s}}+\mathbf{F}_{\mathrm{m}}+\mathrm{mg}=\mathrm{m}^{a} \ddot{\mathbf{P}}_{m}
$$

where ${ }^{a} \ddot{\mathbf{P}}_{m}$ and $\mathbf{g}$ are respectively the acceleration and the gravity. The magnetic force depends on the magnetic field gradient:

$$
\mathbf{F}_{m}=\mathrm{V}(\mathbf{M} \cdot \nabla) \mathbf{B}
$$

considering that the particle is of a volume $\mathrm{V}$ and is made of a ferromagnetic material with a magnetization $\mathbf{M}$. As the particle is assumed saturated, the particle magnetization $\mathbf{M}$ is constant. Hence, the magnetic force is proportional to the applied magnetic field gradient.

The magnetic field, thus the magnetic field gradient, depends on the relative particle position to the electromagnet and the current I applied to this coil. To determine the particle position according to a coil ${ }_{i},{ }^{i} \mathbf{P}_{m}=\left[{ }^{i} x_{m}{ }^{i} y_{m}\right]$, a twist transformation matrix ${ }^{a} \mathrm{~T}_{i}$ is used. In this work, the magnetic field is considered predominant in the coil axis and directed toward it, as the electromagnets are four times the size of the arena and are arranged in a configuration close to the workspace. The magnetic field is then assumed to be varying only in the coil axis, and can be expressed by:

$$
\|\mathbf{B}\|=f_{\mathbf{B}}\left(\mathrm{I},{ }^{i} x_{m}\right)
$$

and by differentiating the magnetic field gradient magnitude is expressed by:

$$
\|\nabla \mathbf{B}\|=f_{\nabla \mathbf{B}}\left(\mathrm{I},{ }^{i} x_{m}\right)
$$

The relationship between the generated magnetic field and the applied current and distance to the coil is interpolated from experimental measurements.

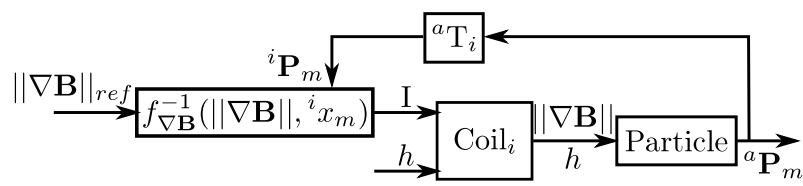

Fig. 3: To apply a given $\|\nabla \mathbf{B}\|_{\text {ref }}$ during a time $h$, the required current $\mathrm{I}$ is computed according to the current particle position to the actuated coil $_{i}$.

In addition, the magnetic force depends on the particle position and the applied current. For a desired displacement, the applied force should be constant regardless of the particle position. As shown in Fig. 3, the system inputs are $\|\nabla \mathbf{B}\|[\mathrm{mT} / \mathrm{mm}]$ and $h[\mathrm{~ms}]$, and produce the movement of the particle at the new position ${ }^{a} \mathbf{P}_{m}$.

\section{Actuation results}

In order to evaluate the magnetic actuation of a particle in contact with a surface, the particle is placed on a nonprocessed glass surface, then is actuated with magnetic forces. The experiment shows that for a given system input $(\|\nabla \mathbf{B}\|, h)$, the output displacement can be null or of different magnitudes. To quantify the system response variability, the following experiment is iterated during 6 days. The daily experiment consists in measuring the displacement of the particle for several inputs. The inputs are default values of the used magnetic platform [19]. $\|\nabla \mathbf{B}\|$ varies from $4 \mathrm{mT} / \mathrm{mm}$ to $8 \mathrm{mT} / \mathrm{mm}$ with a $2 \mathrm{mT} / \mathrm{mm}$ step, $h$ varies from $1 \mathrm{~ms}$ to $5 \mathrm{~ms}$ with a $2 \mathrm{~ms}$ step. For each possible combination $(\|\nabla \mathbf{B}\|, h)$, the signal is applied 80 times during the daily experiment. The occurrence of displacement is recorded if it is non null.
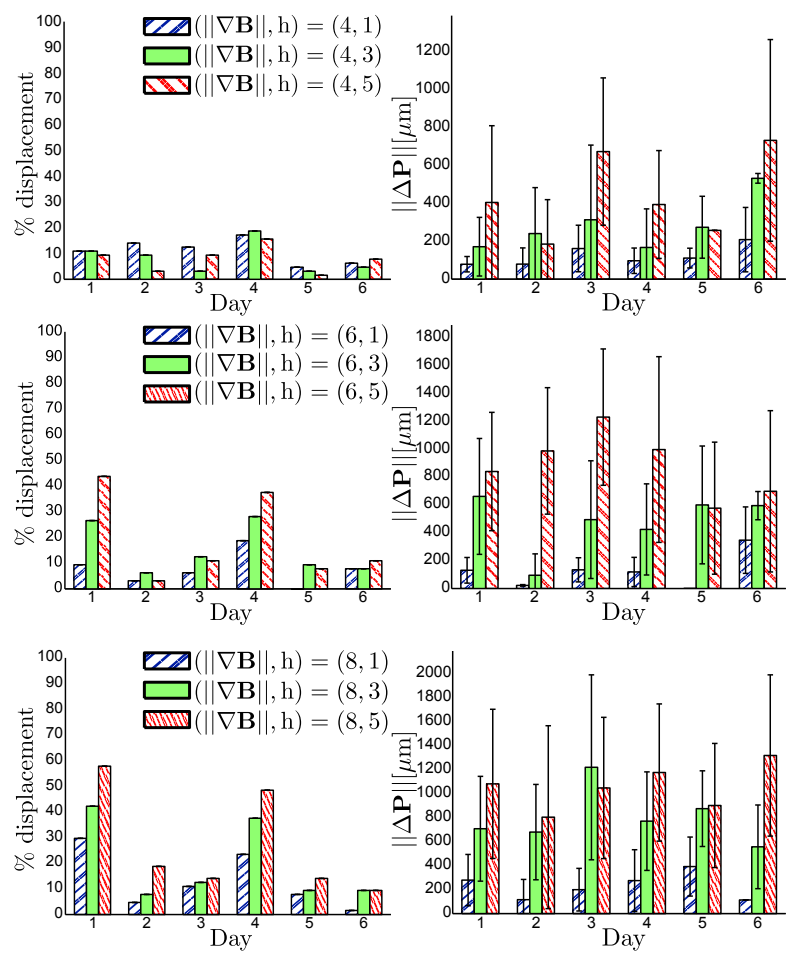

Fig. 4: Variation of the displacement magnitudes depending on the day. The histograms on the left represent the displacement percentages, while those on the right column show the average displacement and standard deviations.

Fig. 4 represents the variation of the displacement percentage over 80 runs for each input $(\|\nabla \mathbf{B}\|, h)$ and the average of the magnitude displacement as a function of the days. The displacement percentage varies daily because of the environment disturbances such as the humidity ratio, temperature, electrostatic forces, etc. However, the displacement percentage increases with the gradient $\|\nabla \mathbf{B}\|$ magnitude, while the magnitude of displacement increases with the signal width $h$. As a conclusion, the response of the particle on a non-processed surface is variable. Such surfaces do not guarantee enough repeatability for closed loop servoing.

\section{SURFACE FORCES REDUCTION}

Surfaces of various roughness and material are used to determine the effect of the surface properties on the surface forces and to limit them.

\section{A. Surface processing}

The surface forces depends on the surface texture and the electrostatics effects. In order to determine the suitable 
substrate for the micro-particles movement, different roughnesses and material are experimented. Surfaces with different roughnesses are produced using micro-fabrication processes in the clean room. The roughness depends on the surface textures, thus, micro patterns are embedded on a silicon substrate. The shape and the size of the pattern as well as the distance between the micro motifs influence the roughness. Fig. 5(a) shows a surface with a $8 \mu \mathrm{m}$ square pattern and a $7 \mu \mathrm{m}$ distance between each pattern.

The process used to produce such surfaces, depicted in Fig. 5(b), consists in lithography and DRIE (deep reactive ion etching) processes. The photolithography is done as follow: first, a photoresistant resin is deposited onto a $\mathrm{Si}$ wafer by spin coating, then the wafer is exposed to UV light through a mask with the desired patterns, followed by the development stage that removes the exposed resin. The DRIE, based plasma etching, is used to etch $11 \mu \mathrm{m}$ deep motifs in the surface. At last, the remaining resin is cleared away by a solvent.

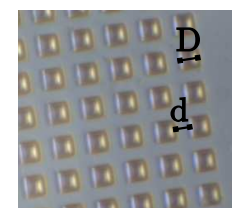

(a) Sample of a controlled surface, $\mathrm{D}$ - is the motif size and -d- is the inter-motifs distance.

Fig. 5: Sample of a surface with an embedded square shaped pattern, the squares length is $8 \mu \mathrm{m}$ and the distance between the patterns is $7 \mu \mathrm{m}$ (a), produced by a micro-fabrication process (b).

To evaluate the effect of the roughness and the surface conductivity on the micro-particle behavior, different surfaces, described in Tab. I, are experimented. Four semi conductive surfaces $\mathrm{Si}$, are tested with a $295.7 \times 410 \mu \mathrm{m}^{2}$ sized particle. A non-conductive glass surface, the reference in this study, is tested with a $300 \times 470 \mu \mathrm{m}^{2}$ sized particle. The surfaces roughness is quantified by the geometric mean roughness -Rq-: the roughness root mean square profile ordinates.

TABLE I: Parameters of the five experienced surfaces.

\begin{tabular}{|c|c|c|c|c|c|}
\hline Surface & I & II & III & IV & Reference \\
\hline Motif shape & Circle & Square & Square & - & - \\
\hline $\mathrm{D}[\mu \mathrm{m}]$ & 25 & 12 & 5 & - & - \\
\hline Depth $[\mu \mathrm{m}]$ & 11 & 11 & 11 & - & - \\
\hline $\mathrm{d}[\mu \mathrm{m}]$ & 5 & 8 & 15 & - & - \\
\hline Material & \multicolumn{7}{|c|}{ Si } & Polished Si & Glass \\
\hline Properties & 4.1 & 5.4 & 4.8 & $0.8^{*} 10^{-3}$ & $0.3^{*} 10^{-3}$ \\
\hline $\operatorname{Rq}[\mu \mathrm{m}]$ & 4 & & \\
\hline
\end{tabular}

\section{B. Actuation results on processed surfaces}

As for the evaluation of the actuation method, the displacement due to a signal input $\|\nabla \mathbf{B}\|$ during a time $h$ is measured 150 times for each input values: $\|\nabla \mathbf{B}\|$ varying from $4 \mathrm{mT} / \mathrm{mm}$ to $8 \mathrm{mT} / \mathrm{mm}$ by $2 \mathrm{mT} / \mathrm{mm}$ step, $h$ varying from $1 \mathrm{~ms}$ to $5 \mathrm{~ms}$ by $2 \mathrm{~ms}$ step. The occurrence of the displacement means that it is not null.

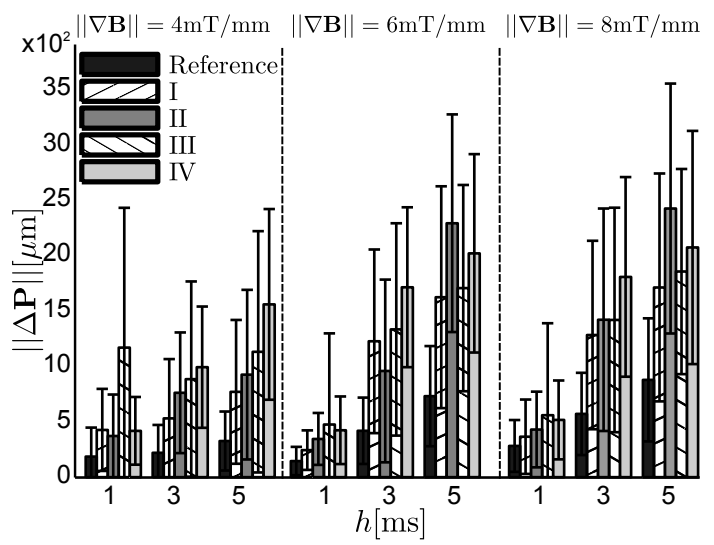

(a) Variations of the averaged displacement magnitudes according to the input signals $\|\nabla \mathbf{B}\|$ and $h$.

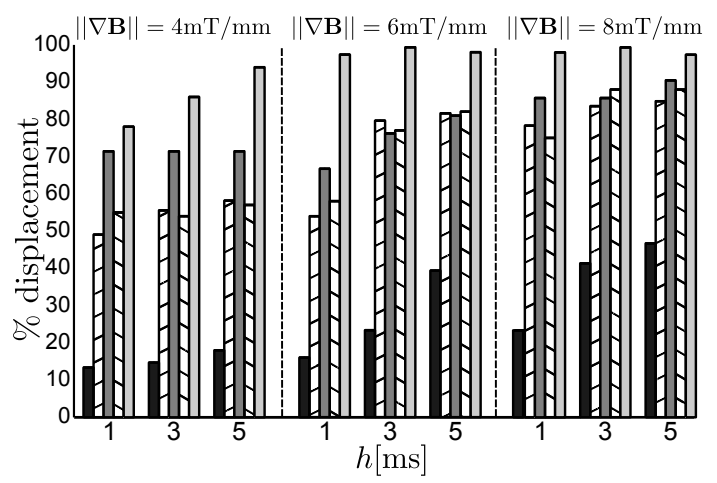

(b) Variation of the displacement occurrence percentages according to the input signals $\|\nabla \mathbf{B}\|$ and $h$.

Fig. 6: Effect of surface roughness and material on the particle displacement percentage and magnitude.

As shown in Fig. 6(b), the percentage of displacement increases with the intensity of the applied magnetic field gradient. It improves as the roughness decreases. Besides, the substrate electrical conductivity influences the adhesion forces. The glass, an insulator, produces the lowest displacement percentage while the $\mathrm{Si}$, a semiconductor, is more adequate for a micro-particle motion. The polished Si surface (surface IV) presents a reduced roughness and electrical resistivity. It guarantees the highest percentage of particle displacements among the tested surfaces. In addition, the averaged displacement magnitudes, in Fig. 6(a), increase with the time $h$ of the applied signal. Although, the standard deviations of the displacement magnitude are high (approximately the averaged displacement magnitude) on all of the experimented surfaces. The processed surfaces improve the occurrence rate of the particle displacement without increasing the particle travel repeatability. The actuation 
repeatability issue can be addressed by an appropriate control law. The particle position, detected by image processing, is used in the next section as a closed loop feedback to correct the particle drift.

\section{LINEAR CONTROL}

To improve the particle repeatability on the surface IV, a closed loop control is used. Thus the identification of the particle response to an input signal is required. The identified model, beside the camera feedback, is used to correct the particle position.

\section{A. Closed loop}

To move the particle from its starting position ${ }^{a} \mathbf{P}_{1}$ to a targeted position ${ }^{a} \mathbf{P}_{2}$, the applied magnetic field gradient $\|\nabla \mathbf{B}\|$ at ${ }^{a} \mathbf{P}_{1}$ during a time $h$ needs to generate the displacement $\|\Delta \mathbf{P}\|=\mid{ }^{a} \mathbf{P}_{2}-{ }^{a} \mathbf{P}_{1} \|$. Thus, the control parameters are: $\|\nabla \mathbf{B}\|, h$. To simplify the system, only one parameter is considered at a time, i.e $h$ remains constant while $\|\nabla \mathbf{B}\|$ is controlled or vice versa. In order to define the fixed parameter $\left\|\nabla \mathbf{B}_{\text {cst }}\right\|$ or $h_{c s t}$, the displacement magnitude and the displacement percentage are considered. Actually, to guarantee a high resolution positioning ( $1 \%$ to $20 \%$ of the particle dimension), the displacement magnitude needs to be small enough. While the displacement percentage needs to be superior to $95 \%$ in order to cancel the system disturbances. These discrimination criteria are applied on a set of experiments. The displacement magnitude and percentage are computed over 150 runs for each input signal $(\|\nabla \mathbf{B}\|$, $h$ ), where $\|\nabla \mathbf{B}\|$ varies from $1 \mathrm{mT} / \mathrm{mm}$ to $5 \mathrm{mT} / \mathrm{mm}$ with a $1 \mathrm{mT} / \mathrm{mm}$ step and $h$ varies from $1 \mathrm{~ms}$ to $5 \mathrm{~ms}$ with a $1 \mathrm{~ms}$ step.
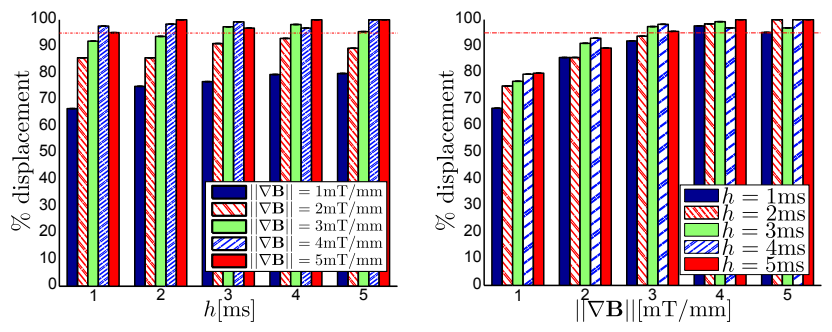

(a) Displacement percentage with (b) Displacement percentage with respect to $h$.

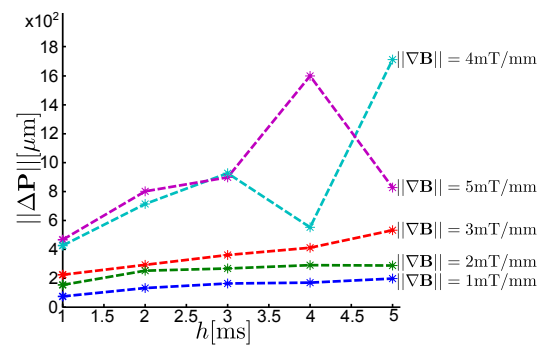

(c) Average magnitude displacement with respect on $h$

Fig. 7: The percentage and average magnitude of displacement is evaluated to define which parameter to keep constant while controlling the other one.
Fig. 7(a) shows that no value of $h$ guarantees that the particle will move with a chance higher than $95 \%$, no matter what the magnetic field gradient intensity is. Thus, a control law based on the time $h$ command is impracticable. However, Fig. 7 (b) shows that both $\|\nabla \mathbf{B}\|=4 \mathrm{mT} / \mathrm{mm}$ and $\|\nabla \mathbf{B}\|=5 \mathrm{mT} / \mathrm{mm}$ assure a displacement percentage greater than $95 \%$ regardless of the time $h$. Moreover, the displacements induced by an applied signal of $\|\nabla \mathbf{B}\|=4 \mathrm{mT} / \mathrm{mm}$ are shorter than the displacements produced by a $\|\nabla \mathbf{B}\|=5 \mathrm{mT} / \mathrm{mm}$. Thus, the suitable magnetic field gradient to produce short displacement, in Fig. 7(c), is $\left\|\nabla \mathbf{B}_{c s t}\right\|=4 \mathrm{mT} / \mathrm{mm}$. In the following, the control law is based on $h$ with a constant magnetic field gradient $\left\|\nabla \mathbf{B}_{\text {cst }}\right\|=4 \mathrm{mT} / \mathrm{mm}$.

The particle position is detected by processing the camera image in the arena frame reference and is converted to the coil $_{i}$ frame of reference using the twist transformation matrix ${ }^{a} \mathrm{~T}_{i}$. The response of the particle can be expressed by the relation $\|\Delta \mathbf{P}\|=g_{h}(h)$. To minimize the error between the measured position and the desired position, the required signal $\left(\left\|\nabla \mathbf{B}_{c s t}\right\|, h\right)$ is defined by inverting the response model $h=g_{h}^{-1}(\|\Delta \mathbf{P}\|)$. Besides, a proportional term, $\mathrm{K}_{p}$, is used to regulate the control loop. The parametrization of the closed loop system, Fig. 8, relies on the characterization of the response model $g_{h}(h)$ and the proportional term.

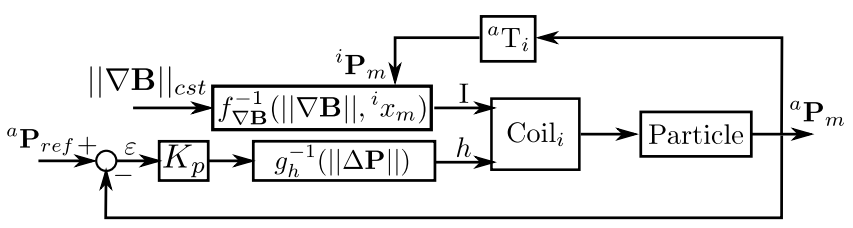

Fig. 8: Closed loop system.

\section{B. Identification of the micro-particle response}

Considering the variability of the displacement magnitude a statistical approach is used to define the response model, $g_{h}$. The displacement of the particle is measured 150 times for each input signal $(\|\nabla \mathbf{B}\|, h)$, and the average magnitude is considered. The linear control requires linear $g_{h}$ law. Thus, these relations are determined by a first order interpolation of the displacement variation according to $\left(\left\|\nabla \mathbf{B}_{c s t}\right\|, h\right)$ :

$$
\|\Delta \mathbf{P}\|=g_{h}(h) \Leftrightarrow\|\Delta \mathbf{P}\|=\alpha_{h} h+\beta_{h}
$$

In this case for a constant $\left\|\nabla \mathbf{B}_{\text {cst }}\right\|=4 \mathrm{mT} / \mathrm{mm}$, the average displacement according to $h$ is interpolated by a first order law and the outliers data are rejected, as shown in Fig. 9.

The proportional term, $\mathrm{K}_{p}$, is estimated experimentally. Currently, the displacement of the particle is produced by a sequential actuation of the coils. To displace the particle from a position $\mathbf{P}_{1}$ to a position $\mathbf{P}_{2}$, by convention the first coil to be actuated is in the direction $\mathbf{x}_{a}$ or $\mathbf{y}_{a}$ where the distance between the initial and the final position is the longest.

\section{Linear control results}

In order to evaluate the positioning performance, the particle is controlled from a start position to a goal position both randomly chosen. This test is repeated 150 times. The 


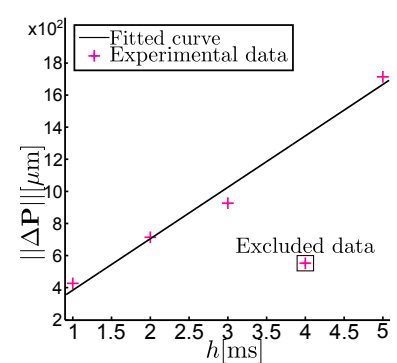

Fig. 9: Fit of the experimental data with a first order interpolation.

time of execution $(t[\mathrm{~s}])$ and the final error $(\| \varepsilon||[\mu \mathrm{m}])$ are measured. A run is considered successful if the positioning is precise at $24 \mu \mathrm{m}, 39 \mu \mathrm{m}$ or $53 \mu \mathrm{m}$ according to the desired precision $6.75 \%, 11 \%$ or $15 \%$ of the particle size, and the execution time does not exceed 12s. The results are summarized in Tab. II.

\begin{tabular}{|c|c|c|c|c|c|}
\hline & & \multicolumn{2}{|c|}{$t[\mathrm{~s}]$} & \multicolumn{2}{|c|}{$\|\varepsilon\|[\mu \mathrm{m}]$} \\
\hline Precision \% & Success rate $\%$ & $\bar{t}$ & $\sigma_{t}$ & $\overline{\|\varepsilon\|}$ & $\sigma_{\|\varepsilon\|}$ \\
\hline 6,75 & 90 & 3,4 & 2,6 & 11,84 & 8,24 \\
\hline 11 & 100 & 2,35 & 1,94 & 29,01 & 9,11 \\
\hline 15 & 100 & 1,88 & 1,56 & 35,44 & 14,01 \\
\hline
\end{tabular}

TABLE II: Summary of the linear control for three precision values over 150 positioning for each precision. $\bar{t}$ and $\overline{\|\varepsilon\|}$ are the mean values and $\sigma_{t}$ and $\sigma_{|| \varepsilon||}$ are the standard deviation of, respectively, the execution times and the final errors.

The positioning of a ferromagnetic micro-particle with a linear control law presents good performance in terms of success rate and precision. However, the execution times are high (average of $1.56 \mathrm{~s}$ for the lowest precision). Besides, the success rate decreases when the required precision is increased (90\% for a precision of $6.75 \%$ of the particle), as well as for the execution times (an average of $3.4 \mathrm{~s}$ and $1.56 \mathrm{~s}$ respectively for $15 \%$ and $6,75 \%$ of precision). A proportional corrector is enough to a fast approach of the reference position, but the control needs to be improved for a more precise positioning, and a reliable trajectory tracking.

\section{CONCLUSIONS}

This work addresses the magnetically actuated microdevices in a dry environment. The main challenge is the surface adhesion particularly predominant in the dry environment. The proposed method to limit the surface forces relies on the processing of the workspace substrate. The experiments on multiple material and roughness surfaces show that the reduced roughness and electrical resistivity of the surface improves the particle displacement probability. Besides, to control the particle position, a closed loop system is required. The particle response model is identified using a statical approach. The positioning of the particle is achieved, on a flat and controlled surface, by a simple linear control law. The control law is reliable at $90 \%$ for a precision of
$6.75 \%$ of the controlled particle size and is $100 \%$ reliable for lower precision.

In addition, in this work the control of the particle orientation is not considered. For further improvement of the system the simultaneous actuation of the coils can be used to control the particle orientation beside the position, thus, to perform trajectory tracking at high precision.

\section{REFERENCES}

[1] M. Hagiwara, T. Kawahara, T. Iijima, Y. Yamanishi, and F. Arai. High speed microrobot actuation in a microfluidic chip by levitated structure with riblet surface. ICRA, pages 2517-2522, 2012.

[2] F. Ullrich, C. Bergeles, J. Pokki, O. Ergeneman, S. Erni, G. Chatzipirpiridis, S. Pané, C. Framme, and B. J. Nelson. Mobility experiments with microrobots for minimally invasive intraocular surgery. IOVS, pages 2853-63, 2013.

[3] I. S. M. Khalil, R. M. P. Metz, L. Abelmann, and S. Misra. Interaction force estimation during manipulation of microparticles. IROS, pages 950-956, 2012.

[4] B. R. Donald, C. G. Levey, C. D. Mcgray, I. Paprotny, and D. Rus. An untethered, electrostatic, globally controllable mems micro-robot. J. Microelectromech. Syst., pages 1-15, 2006.

[5] M. Kharboutly and M. Gauthier. High speed closed loop control of a dielectrophoresis-based system. ICRA, pages 1438-1443, 2013.

[6] S. Sakuma and F. Arai. Cellular force measurement using a nanometric-probe-integrated microfluidic chip with a displacement reduction mechanism. Journal of Robotics and Mechatronics, pages 277-284, 2013.

[7] T. Xu, G. Hwang, N. Andreff, and S. Régnier. Modeling and swimming property characterizations of scaled-up helical microswimmers. IEEE/ASME Transactions on Mechatronics, pages 1-11, 2013.

[8] B. J. Nelson, I. K. Kaliakatsos, and J. J. Abbott. Microrobots for minimally invasive medicine. Annual Review of Biomedical Engineering, pages 55-85, 2010.

[9] A. W. Mahoneya, J. C. Sarrazinb, E. Bambergb, and J. J. Abbott. Velocity control with gravity compensation for magnetic helical microswimmers. Advanced Robotics, pages 1007-1028, 2011.

[10] I. S. M. Khalil, F. van den Brink, O. S. Sukas, and S. Misra. Microassembly using a cluster of paramagnetic microparticles. ICRA, pages 5527-5532, 2013.

[11] E. Diller, J. Giltinan, and M. Sitti. Independent control of multiple magnetic microrobots in three dimensions. Int. J. Rob. Res., pages 614-631, 2013.

[12] S. Schuerle, S. Erni, M. Flink, B. E. Kratochvil, and B. J. Nelson. Three-dimensional magnetic manipulation of micro- and nanostructures for applications in life sciences. IEEE Transactions on Magnetics, pages 321-330, 2013.

[13] D. Folio, C. Dahmen, T. Wortmann, A. M. Zeeshan, Kaiyu Shou, S. Pané, B. J. Nelson, A. Ferreira, and S. Fatikow. Mri magnetic signature imaging, tracking and navigation for targeted micro/nanocapsule therapeutics. IROS, pages 1297-1303, 2011.

[14] K. Belharet, D. Folio, and A. Ferreira. Three-dimensional controlled motion of a microrobot using magnetic gradients. Advanced Robotics, pages 1069-1083, 2011.

[15] M. Michihata, T. Yoshikane, T. Hayashi, and Y. Takaya. New technique of single-beam gradient-force laser trapping in air condition. ISOT, pages 1-6, 2012.

[16] M. Kummer, J. J. Abbott, B. E. Kratochvil, R. Borer, A. Sengul, and B. J. Nelson. Octomag: An electromagnetic system for 5-dof wireless micromanipulation. IEEE Trans. Rob., pages 1006-1017, 2010.

[17] J.D. Keuning, J. De Vriesy, L. Abelmanny, and S. Misra. Image-based magnetic control of paramagnetic microparticles in water. IROS, pages 421-426, 2011.

[18] D. R. Frutiger, B. E. Kratochvil, K. Vollmers, and B. J. Nelson. Small, fast, and under control: wireless resonant magnetic micro-agents. Int. J. Rob. Res., pages 613-636, 2010.

[19] A. I. Ivan, G. Hwang, J. Agnus, M. Rakotondrabe, N. Chaillet, and S. Régnier. First experiment on magpier : a planar wireless magnetic and piezoelectric microrobot. ICRA, pages 102-108, 2011. 